\title{
Scale-Up of High Shear Granulation Based on Agitation Power
}

\author{
Yoshinobu Sato, * Takumi Oкамото, and Satoru Watano \\ Osaka Prefecture University; Sakai, Osaka 599-8531, Japan. Received June 22, 2005; accepted September 22, 2005
}

\begin{abstract}
Scale-up of wet granulation in a vertical high shear mixer was conducted. Pharmaceutical excipient mixtures composed of lactose, cornstarch and micro-crystalline cellulose, and hydroxypropylecellulose as a binder were mixed together and then granulated with purified water under various operating conditions and vessel scales. Torque of agitator shaft was continuously measured and then agitation power per unit vessel volume was calculated. The agitation power per unit vessel volume showed a good correlation with physical properties of obtained granules, such as mass median diameter, strength and compressibility. This implied that the scale-up characteristics could be well analyzed by means of the agitation power per unit vessel volume. In addition, the effects of agitator tip speed and Froude number on the agitation power per unit vessel volume were investigated. The results showed that the agitation power per unit vessel volume was well characterized by the tip speed rather than the Froude number. This meant that the granule growth mainly progressed by the shear stress from the agitator blade. Dynamic characteristics of high shear granulation were also discussed here.
\end{abstract}

Key words scale-up; granulation; high shear mixer; agitation power; tip speed

Granulation in a high shear mixer is widely used in the pharmaceutical, food, chemical, agriculture and other industries. One of the major reasons is that high shear granulation produces spherical and well-compacted granules in a short operational time and its operation is very simple.

In order to understand the granulation mechanism or monitor the granule growth, a number of investigations have been carried out. So far, Lindberg et al. ${ }^{1,2)}$ reported torque measurement of a main driving shaft by using strain gages. Leuenberger ${ }^{3,4)}$ measured power consumption of motor drive to monitor the operating conditions. Holm et al. $^{5)}$ described the relationship between granule growth and power consumption curves. They also investigated the effect of the process parameters on the granule properties. ${ }^{6-8)}$ Watano et $a{ }^{9}{ }^{9)}$ developed a novel image processing system for direct measurement of granule growth and then controlled the high shear granulation process by a fuzzy logic. ${ }^{10)}$

Despite the various studies on the measurement and control of granule growth, the scale-up of high shear granulation still relies on experiences of expert operators. This is because the granule behavior in a high shear mixer is so complicated that the dynamic characteristics are poorly understood.

As for the scale-up studies, several approaches have been attempted. Landin et al. $^{11)}$ investigated the relationship between wet mass properties and power consumption using different sizes of high shear granulator. The application of experimental design to scale-up of high shear granulation was described by Vojnovic et al. ${ }^{12)}$ However, the dynamic characteristics of granulation in a high shear mixer have not been well studied yet. Therefore, there is still in need of further investigations to understand the scale-up characteristics.

In this study, wet granulation was conducted in vertical high shear mixers of three different vessel scales. Torque of agitator shaft was continuously monitored during the granulation operations and the physical properties of granules, such as mass median diameter, strength and compressibility, prepared under various operating conditions were evaluated. The scale-up characteristics were then discussed based on the obtained results. In addition, agitation power was analyzed to investigate the dynamic characteristics of the high shear granulation.

\section{Experimental}

Apparatus Figure 1 shows a schematic diagram of the experimental apparatus. Vertical high shear mixers with three different vessel scales (SPG25, 200 and 400, Fuji Paudal Co., Ltd.) were used for the scale-up experiments. The dimensions of each scale are listed in Table 1. Each of them obeyed the geometric similarity. The bottom of the vessel was equipped with an agitator blade rotating horizontally, which promoted agglomeration and compaction. A chopper blade was also provided on the side wall of the vessel to break up wet lump into small granules. Torque of the agitator shaft was continuously measured by a digital torque meter and then processed by a personal computer.

Powder Samples Table 2 lists powder samples used. Pharmaceutical excipients composed of lactose, cornstarch and micro-crystalline cellulose were used. The mixed ratio of each component referred to the standard formulation defined by the working group on standard formulation for granulation. ${ }^{13)}$ Hydroxypropylecellulose (HPC-L) was adopted as a binder, which was mixed $3 \mathrm{wt} \%$ (dry basis) in the form of a dry powder into the above excipients before granulation. Purified water was used as a binder solution.

Experimental Procedures Operating conditions for the scale-up experiments are summarized in Table 3. The experiments were conducted as follows. The weighed powder samples were fed into the vessel of granulator

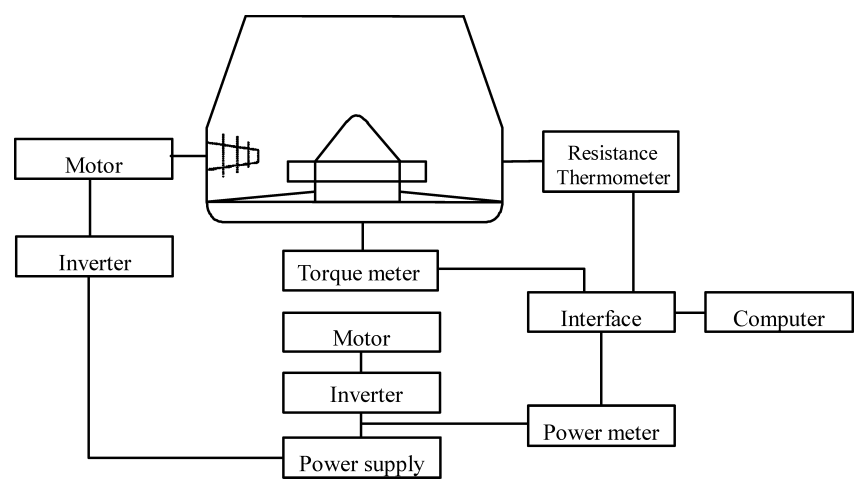

Fig. 1. Schematic Diagram of Experimental Apparatus

Table 1. Dimensions of Vessels

\begin{tabular}{lccc}
\hline \hline & SPG-25 & SPG-200 & SPG-400 \\
\hline Vessel volume [1] & 25.7 & 205.9 & 401.8 \\
Vessel diameter [mm] & 400 & 800 & 1000 \\
Scale ratio [-] & 1.0 & 8.0 & 15.6 \\
Total charge mass [kg] & 5.79 & 46.2 & 90.2 \\
\hline
\end{tabular}


Table 2. Powder Samples

\begin{tabular}{lcc}
\hline \hline \multicolumn{1}{c}{ Samples } & Company and grade & $\begin{array}{c}\text { Charge mass } \\
\text { ratio [\%] }\end{array}$ \\
\hline $\begin{array}{l}\text { Lactose } \\
\text { Cornstarch }\end{array}$ & $\begin{array}{c}\text { DMV (Phaematose 200M) } \\
\text { Nihon Shokuhin Kako Co., Ltd. } \\
\text { (Cornstarch W) }\end{array}$ & 67.2 \\
$\begin{array}{c}\text { Microcrystalline } \\
\text { cellulose } \\
\begin{array}{c}\text { Hydroxypropyle } \\
\text { cellulose } \\
\text { Total }\end{array}\end{array}$ & $\begin{array}{c}\text { Asahi Kasei Chemicals Co., Ltd. } \\
\text { (Avicel PH101) }\end{array}$ & 4.0 \\
Nippon Soda Co., Ltd. (HPC-L) & 3.0 \\
\hline
\end{tabular}

Table 3. Operating Conditions

\begin{tabular}{llll}
\hline \hline & \multicolumn{1}{c}{ SPG-25 } & \multicolumn{1}{c}{ SPG-200 } & SPG-400 \\
\hline Agitator rotational speed [rpm] & $200,300,400$ & $100,150,200$ & $50,100,200$ \\
Chopper rotational speed [rpm] & & 3000 (constant) \\
Moisture content [\%] & & 22.0 (constant) \\
Operating time [min] & & 10 (constant) & \\
& &
\end{tabular}

and mixed for $120 \mathrm{~s}$. The agitator rotational speed was set to run at the prescribed rotational speed, while the chopper rotational speed was set at $3000 \mathrm{rpm}$ constant regardless of the vessel scales. The binder solution (purified water) was added instantaneously from top of the vessel through a stainless funnel, followed by the granulation for $10 \mathrm{~min}$. The ratio of binder solution to powder mass was kept constant throughout all the vessel scales to maintain the same adhesion force. In a high shear granulation, granule growth is considered to be determined by both the adhesion and external forces, i.e. agitation force from the agitator blade. The agitation force is scale-dependent, and has been recognized to greatly influence on the dynamic characteristics of the granulation. In order to analyze the effect of the agitation force from the blade on the dynamic characteristics, the granulation time was fixed constant in this study. The ratio of binder solution to powder mass and the granulation time were decided preliminarily that granules ranging from 75 to 850 um sieve (18 - 200 mesh) could be obtained with a high yield. The obtained granules were dried in a shelf dryer at $50{ }^{\circ} \mathrm{C}$ for $24 \mathrm{~h}$.

Evaluation of Granule Properties Mass median diameter of the obtained granules was determined by a sieve analysis with a rotating sieve shaker. About $100 \mathrm{~g}$ of the granules were shaken for $180 \mathrm{~s}$. After measuring the weight of the granules on each sieve, size distribution was calculated based on a log-normal distribution with a personal computer.

Strength of granules was measured by a strength tester (Grano, Okada Seiko). A granulated particle (diameter range between 300 and 355 um) was placed on a flat adjustment stage to be pressed by a pressing rod, which moved down vertically from above at a constant speed of $100 \mu \mathrm{m} / \mathrm{s}$. The moved displacement and pressed load (force) were measured continuously. The complete breaking load estimated the strength of granulated particle. The strength of granules was finally represented by the average value of the 50 measurements. Compressibility was defined by bulk and tapped densities of the granules as follows:

$$
\text { compressibility }[\%]=\left(\rho_{\mathrm{T}}-\rho_{\mathrm{B}}\right) / \rho_{\mathrm{T}} \times 100
$$

where $\rho_{\mathrm{T}}$ and $\rho_{\mathrm{B}}$ showed tapped and bulk densities of granules $\left[\mathrm{kg} / \mathrm{m}^{3}\right]$, respectively.

\section{Results and Discussion}

Relationship between Agitation Power per Unit Vessel Volume and Granule Properties Power consumption of a motor drive of the main shaft has been widely used for the end-point determination in a high shear granulation. ${ }^{3,4,6)}$ Torque measurement of the agitator blade (main impeller), which requires installation of strain gages on the blade or on the coupling between the motor and the agitator shaft, is also a useful technique for monitoring the process conditions. ${ }^{1,2}$

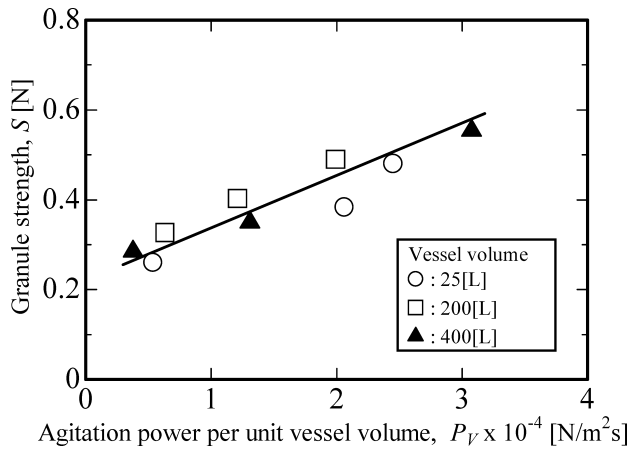

Fig. 2. Relationship between Granule Strength and Agitation Power per Unit Vessel Volume (Correlation Coefficient 0.9320, $n=9$ )

It was also reported that the agitation torque was in good agreement with the power consumption ${ }^{14)}$ and more sensitive than the power consumption due to the direct measurement of the load on the blade. ${ }^{15,16)}$

Agitation power per unit vessel volume, which is calculated from the agitation torque, is a well-known factor that can scale-up the agitation vessel ${ }^{17}$ ) (Eq. 2). It is based on the concept that the same agitation effect can be obtained if the agitation power per unit volume is the same.

$$
P_{V}=\omega T / V
$$

where $T$ indicates agitation torque $[\mathrm{Nm}]$, which is deducted the torque of idling from the one actually measured during the mixing process. $\omega$ and $V$ show angler velocities of the agitator blade and vessel volume, respectively.

Considering the previous studies on the high shear granulation and the concept of the agitation power per unit volume, the agitation power per unit volume was expected to well describe the scale-up characteristics of high shear granulation.

Figure 2 indicates the relationship between the agitation power per unit vessel volume and the granule strength. Seen from the figure, the granule strength increased linearly with the agitation power per unit vessel volume regardless of the vessel scales. With an increase in the agitation power per unit vessel volume, granules received more compaction effect from the agitator blade, leading to have the higher granule strength. The results also confirmed that the same granule strength could be obtained by maintaining the same agitation effect through the agitation power per unit vessel volume.

Figures 3 and 4 investigate the effect of agitation power per unit vessel volume on granule mass median diameter and compressibility, respectively. From these figures, the granule mass median diameter and compressibility were well expressed by the agitation power per unit vessel volume as well. This meant that agglomeration and densification of granules progressed with an increase in the agitation power unit vessel volume. In addition, higher compaction effect from the agitation blade made granules more spherical with smoother surface, which led to the smaller compressibility. From these results, the agitation power per unit vessel volume could well reflect the changes in granule properties and be a useful index for the scale-up of high shear granulation.

Dynamic Characteristic of High Shear Granulation In a high shear mixer, granules receive shear stress from the blade and centrifugal force (inertia force) caused by the agitator rotation, to make the vortex flow. The agitation power 


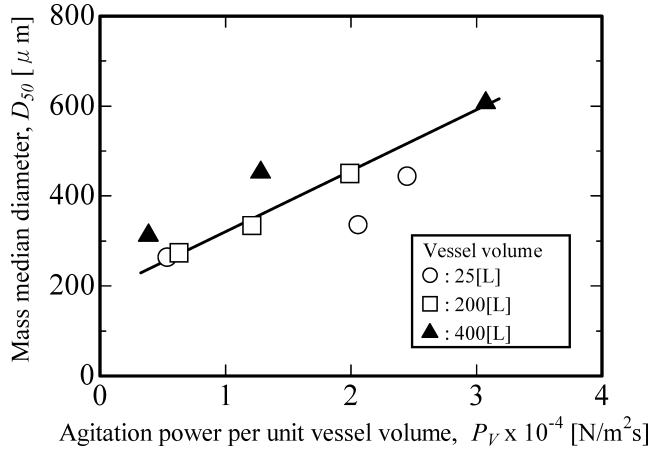

Fig. 3. Relationship between Granule Mass Median Diameter and Agitation Power per Unit Vessel Volume (Correlation Coefficient 0.8472, $n=9$ )

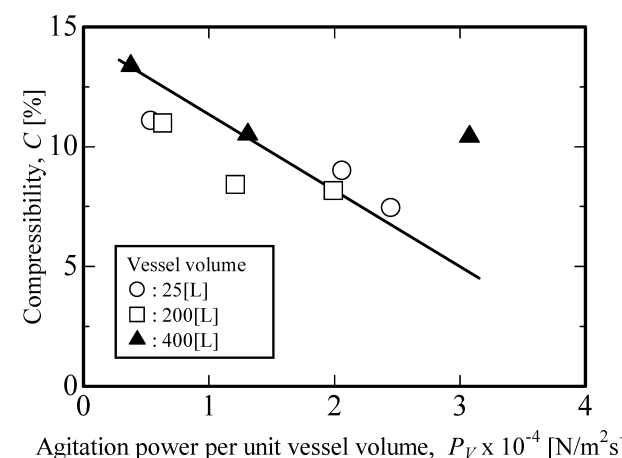

Fig. 4. Relationship between Granule Compressibility and Agitation Power per Unit Vessel Volume (Correlation Coefficient 0.6182, $n=9$ )

arises as a result of the load from granules against the blade, reflecting the changes in granules properties. The shear stress relates to the agitator tip speed, while the centrifugal force can be represented by Froude number (Eq. 3);

$$
\mathrm{Fr}=n^{2} d / g
$$

where $n, d$ and $g$ show agitator rotational speed [1/s], agitator blade diameter $[\mathrm{m}]$, and gravitational acceleration $\left[\mathrm{m} / \mathrm{s}^{2}\right]$, respectively.

In order to analyze the dynamic characteristics of high shear granulation, the effects of the tip speed and the Froude number on the agitation power per unit vessel volume were investigated.

Figures 5 and 6 illustrate the agitation power per unit vessel volume as a function of the tip speed and the Froude number, respectively. As shown in the figures, the tip speed had a linear relationship with the agitation power per unit vessel volume, while less linearity was observed with the Froude number. This indicated that the agitator tip speed had stronger effect on the agitation power per unit vessel volume than the centrifugal force. Watano et al. measured stresses that granules received during high shear granulation by a novel stress measurement system, ${ }^{18)}$ and reported that the stress of tangential direction was much larger than that of normal direction. It was also reported that the tangential stress was shear stress from the blade, while the normal stress had a linear correction with the centrifugal acceleration, and that the shear stress had much stronger effect on granule growth in high shear granulation.

The results obtained here also supported that the shear stress from the blade was closely related to the granule

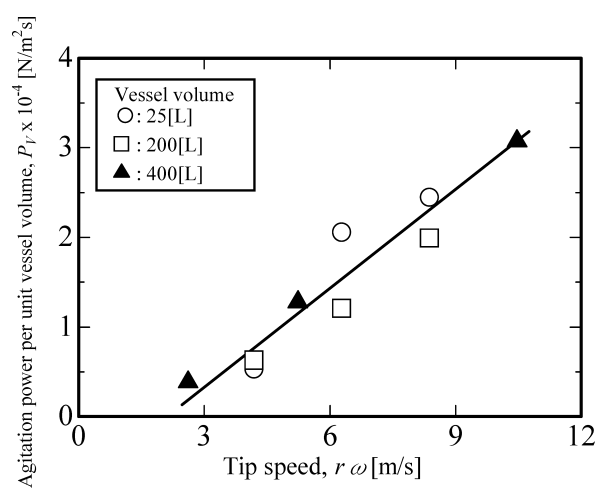

Fig. 5. Agitation Power per Unit Vessel Volume as a Function of Tip Speed (Correlation Coefficient 0.9546, $n=9$ )

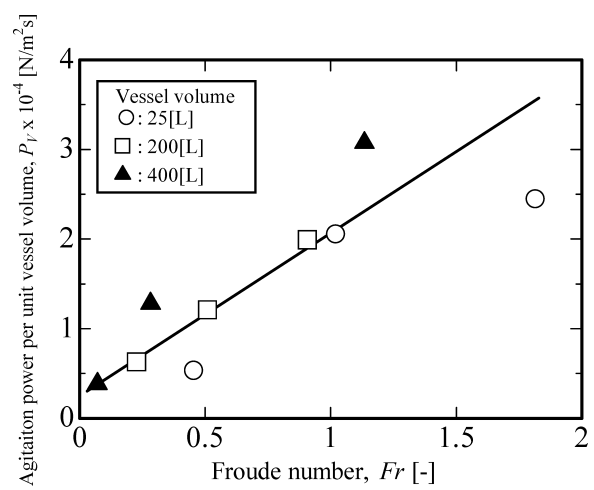

Fig. 6. Agitation Power per Unit Vessel Volume as a Function of Froude Number (Correlation Coefficient $0.8471, n=9$ )

growth. This meant that granule growth in high shear granulation mainly progressed by the shear stress from the blade rather than the impaction force against vessel wall.

\section{Conclusions}

Wet granulation in a vertical high shear mixer was conducted under various operating conditions and vessel scales. Relationships between granule properties (mass median diameter, strength and compressibility) and agitation power per unit vessel volume were investigated. It was found that the agitation power per unit vessel volume had a good correlation with the granule properties and could well express the scale-up characteristics. In addition, the analysis of agitation power per unit vessel volume by agitator tip speed and Froude number indicated that the agitation power per unit vessel volume was well characterized by the tip speed, not by the Froude number. This implied the dynamic characteristics of high shear granulation could be expressed by the shear stress from the agitator blade.

\section{References}

1) Lindberg N. O., Pharm. Acta Helv., 14, 197-204 (1977).

2) Lindberg N. O., Leander L., Reenstierna B., Drug Dev. Ind. Pharm., 8, 775-782 (1982).

3) Leuenberger H., Pharm. Acta Helv., 57, 72-82 (1982).

4) Leuenberger H., Acta Pharm. Technol., 29, 274-280 (1983).

5) Holm P., Schaefer T., Kristensen H. G., Powder Technol., 43, 213 223 (1985).

6) Holm P., Schaefer T., Kristensen H. G., Powder Technol., 43, 225233 (1985).

7) Holm P., Jungersen O., Schaefer T., Kristensen H. G., Pharm. Ind., 45, $806-811(1983)$ 
8) Holm P., Jungersen O., Schaefer T., Kristensen H. G., Pharm. Ind., 46, 97-101 (1984).

9) Watano S., Numa T., Miyanami K., Osako Y., Chem. Pharm. Bull., 48, 1154-1159 (2000).

10) Watano S., Numa T., Miyanami K., Osako Y., Powder Technology, 115, 124-130 (2001).

11) Landin M., York P., Cliff M. J., Rowe R. C., Wigmore A. J., Int. J. Pharmaceut., 133, 127-131 (1996).

12) Vojnovic D., Moneghini M., Rubessa F., Zanchetta A., Drug Dev. Ind. Pharm., 19, 1479-1496 (1993).

13) Sunada H., Pharm. Tech. Jpn., 5, 299-304 (1989).
14) Bier H. P., Leuenberger H., Sucker H., Pharm. Ind., 41, 375-380 (1979).

15) Corvari V., Fry W. C., Seibert W. L., Augsburger L., Pharm. Res., 9, 1525-1533 (1992).

16) Kopcha M., Roland E., Bubb G., Vadino W. A., Drug Dev. Ind. Pharm., 18, 1945-1968 (1992).

17) Perry H. R., Chilton C. H., "Chemical Engineer's Handbook," 5th ed., McGraw-Hill, New York, 1973.

18) Watano S., Okamoto T., Sato Y., Osako Y., Chem. Pharm. Bull., 53, 351-354 (2005) 